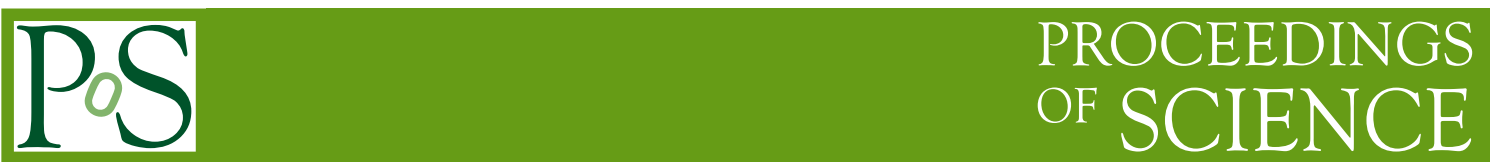

\title{
Hyperons and Neutron Stars
}

\section{Isaac Vidaña}

Centro de Física da Universidade de Coimbra (CFisUC), Department of Physics, University of Coimbra, PT-3004-516 Coimbra, Portugal

E-mail: ividanaefis.uc.pt

In this work I briefly review the role played by hyperons in determining the properties of neutron and proto-neutron stars. In particular, I review the so-called "hyperon puzzle", i.e., the problem of strong softening of the equation of state $(\mathrm{EoS})$ of dense matter due to the presence of hyperons which leads to maximum masses of compact stars that are not compatible with the recent observations of $\sim 2 M_{\odot}$ millisecond pulsars. I discuss some of the solutions that were proposed to tackle this problem. Finally, I re-examine the influence of hyperons on the cooling of newly born neutron stars as well as on the development of the r-mode instability.

The Modern Physics of Compact Stars 2015

30 September 2015 - 3 October 2015

Yerevan, Armenia 


\section{Introduction}

Neutron stars are born in the gravitational collapse of massive stars during a Type-II, Ib or Ic supernova event. Their masses are typically of the order of $1-2 M_{\odot}$, where $M_{\odot} \simeq 2 \times 10^{33} \mathrm{~g}$ is the mass of the Sun, and their radii are within the range $10-12 \mathrm{~km}$. With central densities in the range of $4-8$ times the normal nuclear matter saturation density, $\varepsilon_{0} \sim 2.7 \times 10^{14} \mathrm{~g} / \mathrm{cm}^{3}\left(\rho_{0} \sim 0.16\right.$ $\mathrm{fm}^{-3}$ ), neutron stars are most likely among the densest objects in the Universe [1]. These objects are excellent laboratories to test our present understanding of the theory of strongly interacting matter under extreme conditions. They offer an interesting interplay between nuclear physics of dense matter and astrophysical observations in a borad spectrum of electromagnetic waves.

The conditions under which matter exists inside neutron stars are very different from those one can find in laboratories on Earth. Therefore, a good theoretical knowledge of the equation of state (EoS) of dense matter is required to understand the properties of these objects. Up to date one of the interesting open questions is the state of the matter in the interiors of neutron stars. Traditionally the core of neutron stars has been modeled as a uniform fluid of neutron-rich nuclear matter in equilibrium with respect to the weak interactions ( $\beta$-stable matter). However, due to the large value of the density, new hadronic degrees of freedom are expected to appear in addition to nucleons. Hyperons, baryons with a strangeness content, are an example of these new degrees of freedom. Contrary to terrestrial conditions, where hyperons are unstable and decay into nucleons through weak interactions, matter in neutron stars maintains the weak equilibrium between the decays and their inverse capture processes. Hyperons may appear in the inner cores of neutron stars at densities around $(2-3) \times \rho_{0}$. Their onset leads to a softening of the high-density domain of the EoS and consequently to a reduction of the maximum mass of the stellar sequences constructed from such EoS.

Hyperons can strongly influence not only the masses and radii of neutron stars, but also their thermal evolution and gravitational instabilities. These aspects of neutron star physics are very sensitive to their composition, and therefore to the hyperonic content of neutron star interiors. The cooling of neutron stars is affected by the presence of hyperons because they modify neutrino emissivity of dense matter and allow for fast cooling mechanisms. Furthermore, the emission of gravitational waves in hot and rapidly rotating neutron stars due to the so-called r-mode instability is affected by hyperons, because hyperons dominate the bulk viscosity of matter as soon as they appear in the neutron star interior.

The remainder of this review is organized as follows. In Sec. 2 I discuss in some detail the hyperon puzzle and a variety of possible solutions proposed during the recent years. Section 3 is devoted to the cooling of hyperonic stars, where I discuss the role played by hyperons in determining the emissivity of newly born neutron stars. In Sec. 4 I discuss the physics of damping of r-mode instability and the contribution of hyperons to this damping. I close this review with a summary in Sec. 5

\section{The hyperon puzzle}

The presence of hyperon in neutron stars was considered for the first time in the pioneering work of Ambartsumyan and Saakyan in 1960 [2]. Since then, their effects on the properties of 

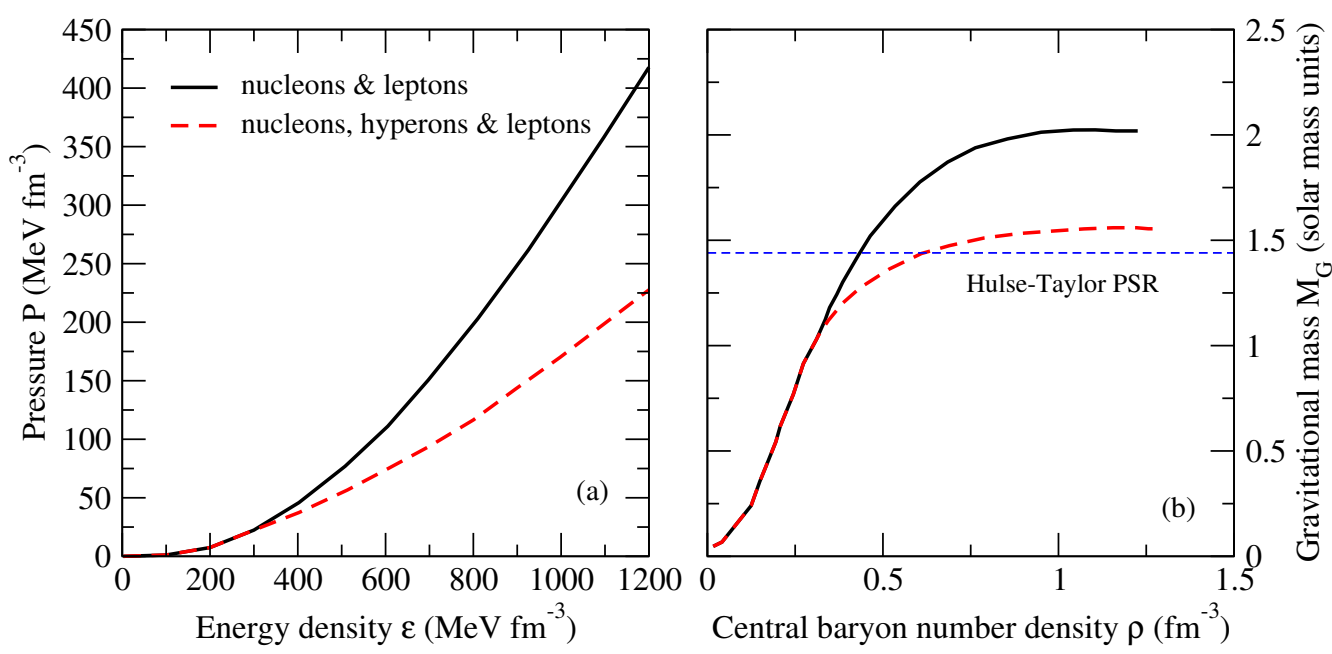

Figure 1: Illustration of the effect of the presence of hyperons on the EoS (panel (a)) and mass of a neutron star (panel (b)). A generic model with (black solid line) and without (red dashed line) hyperons has been considered. The horizontal line shows the observational mass of the Hulse-Taylor [14] pulsar.

these objects have been studied by many authors using either phenomenological $[3,4]$ or microscopic $[5,6,7,8,9]$ approaches to the EoS of neutron star matter. Phenomenological approaches, either relativistic or non-relativistic, are based on effective density-dependent interactions which typically contain a certain number of parameters adjusted to reproduce nuclear and hypernuclear observables, and neutron star properties. Relativistic mean field (RMF) models [3] and models based on the Skyrme-type interactions [4] are among the most commonly used ones. Microscopic approaches, on the other hand, are based on realistic two-body baryon-baryon interactions that describe the scattering data in free space. These realistic interactions have been mainly constructed within the framework of a meson-exchange theory [10,11], although recently a new approach based on chiral perturbation theory has emerged as a powerful tool [12]. In order to obtain the EoS one has to solve then a very complicated many-body problem [13]. A great difficulty of this problem lies in the treatment of the repulsive core, which dominates the short-range behavior of the interaction. Although many different microscopic many-body methods have been extensively used to study nuclear matter, very few of these have been extended to hypernuclear sector. To my knowledge, these many-body methods include the Brueckner-Hartree-Fock (BHF) approximation [5] of the Brueckner-Bethe-Goldstone theory, the Hartree-Fock theory based on the soft $V_{\text {lowk }}$ interactions [6] and the Dirac-Brueckner-Hartree-Fock theory [7, 8]. Very recently the Auxiliary Field Diffusion Monte Carlo method [9] was also extended to the hyperonic sector.

All these approaches agree that hyperons may appear in the inner core of neutron stars at densities around $\sim(2-3) \times \rho_{0}$. At such densities, the neutron chemical potential is large enough to make the conversion of a neutron into hyperon energetically favorable. This conversion relieves the Fermi pressure exerted by nucleons and makes the EoS softer, which is illustrated in panel (a) of Fig. 1 for a generic model with (black solid line) and without (red dashed line) hyperons. As a consequence (see panel (b)) the masses of the stars with central densities beyond the onset density of the hyperons are lower and the maximum mass of hyperonic stars is substantially reduced. In 
microscopic calculations (see e.g., Refs. [5, 6]), the reduction of the maximum mass can be even below the "canonical" one of $1.4-1.5 M_{\odot}[14]$. This is not the case, however, in phenomenological calculations which find maximum masses compatible with the canonical value above. In fact, most relativistic models including hyperons predict maximum masses in the range $1.4-1.8 M_{\odot}[3]$.

Although the presence of hyperons in neutron stars seems to be energetically unavoidable, the strong softening of the EoS associated with the onset of hyperons (notably in microscopic models) leads to maximum masses not compatible with observations. This controversy is known as hyperon (or hyperonization) puzzle, which is a subject of current intensive research. The discrepancy between the theory and observations became more dramatic after the recent measurements of unusually high masses of the millisecond pulsars PSR J1903+0327 (1.667 \pm 0.021$)$ [15], PSR J1614-2230 (1.97 $\left.\pm 0.04 M_{\odot}\right)[16]$, and PSR J0348+0432 $\left(2.01 \pm 0.04 M_{\odot}\right)$ [17] which rule out almost all currently proposed EoS with hyperons (both microscopic and phenomenological).

To solve this problem a mechanism is needed that could provide the additional repulsion that makes the EoS stiffer and, therefore, the maximum mass compatible with the current observational limits. Three different mechanisms that could provide such additional repulsion have been proposed: (i) more repulsive hyperon-hyperon interactions in relativistic density functional methods driven by either repulsive vector mesons exchanges [18, 19, 20, 21] or less-attractive scalar $\sigma$ meson exchange [22] (ii) repulsive hyperonic three-body forces [23, 24, 25, 26] and (iii) phase transition to deconfined quark matter at densities below the hyperon threshold [30, 31, 32, 33, 34]. The remainder of this Section contains a discussion of each of these solutions which is followed by a comment on the possible roles played by the $\Delta$ isobar and kaon condensation in neutron stars.

\subsection{Hyperon-hyperon repulsion}

This solution, which has been mainly explored in the context of RMF models (see e.g., Refs. $[18,19,20,21,22])$, replies on the well-known fact that in meson-exchange models of nuclear forces vector mesons generate repulsion at short distances while $\sigma$-meson is responsible for intermediate range attraction. If the interaction of hyperons with vector mesons is repulsive enough or the attraction driven by $\sigma$-meson is weak enough the EoS could be sufficiently stiff to explain the current pulsar mass observations. The modifications of the strength of meson exchanges must be consistent with the hypernuclear data which requires, at least, the $\Lambda \mathrm{N}$ interaction to be attractive and suitable tuned to the hypernuclear data [35]. Such tuning is not required if the repulsive vector meson interactions acts only among the hyperons through the exchange of the strange $\phi$ vector meson (which couples only to hyperons). In this way, the onset of hyperons is shifted to higher densities and neutron stars with maximum masses larger than $2 M_{\odot}$ and a significant hyperon fraction can be successfully obtained. However, it is also possible to tune the interactions of non-strange mesons to accommodate the hypernuclear data and astrophysical constraints on maximum masses of neutron stars in the framework of relativistic density functional theories (for such an approach see e.g. Ref. [22]). For more details the interested reader is referred to the original recent work quoted above.

\subsection{Hyperonic three-body forces}

It is well known that the three-nucleon forces in the nuclear Hamiltonian are fundamental ingredients that are needed to reproduce properly the properties of few-nucleon systems as well as the 
empirical saturation point of symmetric nuclear matter in non-relativistic many-body approaches. Therefore, it seems natural to suggest that three-body forces involving one or more hyperons (i.e., NNY, NYY and YYY) could provide additional repulsion (already established in the case of threenucleon forces) that can solve the hyperon puzzle. Indeed if three-body forces involving hyperons are repulsive enough they can make the EoS stiffer at high densities and, therefore, make the maximum mass of the star compatible with the recent observations. This idea was suggested even before the observation of neutron stars with $\sim 2 M_{\odot}$ (see e.g., Ref. [23]), and it has been explored by a number of authors in the last years [24, 25, 26]. However, no general consensus has been reached regarding the role played by the hyperonic three-body forces in solving the hyperon puzzle. Refs. $[23,25]$ find that the inclusion of hyperonic three-body forces is sufficient to obtain hyperon stars with masses of the order of $2 M_{\odot}$. Ref. [24] finds that the largest maximum mass attainable within their model is $1.6 M_{\odot}$. The results of Ref. [26] are not conclusive enough because they strongly depend on the $\Lambda \mathrm{NN}$ force employed. To conclude, it seems that hyperonic three-body forces offer an interesting microscopic solution to the hyperon puzzle, however the uncertainties involved in the physics of hyperonic three-body forces are too large at the moment to make a definite conclusion possible.

\subsection{Quarks in neutron stars}

Several authors have suggested that an early phase transition from hadronic mater to deconfined quark matter at densities below the hyperon threshold could provide a solution to the hyperon puzzle. Therefore, massive neutron stars could actually be hybrid stars with a stiff quark matter core. The question that arises then is whether quarks can provide sufficient repulsion to produce a $2 M_{\odot}$ neutron star. To yield maximum masses larger than $2 M_{\odot}$, quark matter should have two important and necessary features: (i) a significant overall quark-quark repulsion to maintain stiff EoS, for example, in vector channels and (ii) a strong enough attraction in certain channels which leads to color superconductivity needed to make the deconfined quark matter phase energetically favorable over the hadronic one [36]. Several models of hybrid stars with the necessary properties to generate $2 M_{\odot}$ neutron stars have been proposed [27, 28, 29, 30, 31, 32, 33, 34]. Conversely, the observation of $2 M_{\odot}$ neutron stars may also helped to impose important constraints on the models of hybrid and strange stars with a quark matter core, and improve our present understanding of the hadron-quark phase transition.

\section{4 $\Delta$ isobar and kaon condensation in neutron stars}

An alternative way to circumvent the hyperon puzzle is to invoke the appearance of other hadronic degrees of freedom such as for instance the $\Delta$ isobar or meson condensates that shift the onset of hyperons to higher densities.

The $\Delta$ isobar is often neglected in the studies of neutron stars because its threshold density was found to be higher than the typical densities prevalent in cores of neutron stars. Nevertheless, it has been recently shown by Drago et al., [37] that the onset of the $\Delta$ depends crucially on the density-dependence of the slope of the nuclear symmetry energy, $i$. e., the parameter $L=3 \rho_{0}\left(\partial E_{s y m}(\rho) / \partial \rho\right)_{\rho_{0}}$. By using a state-of-the-art EoS and recent experimental constraints on $L$, these authors showed that the $\Delta$ isobar could actually appear before the hyperons in neutron star interiors. However, they found also that, as soon as the $\Delta$ is present the EoS, as in the case of 

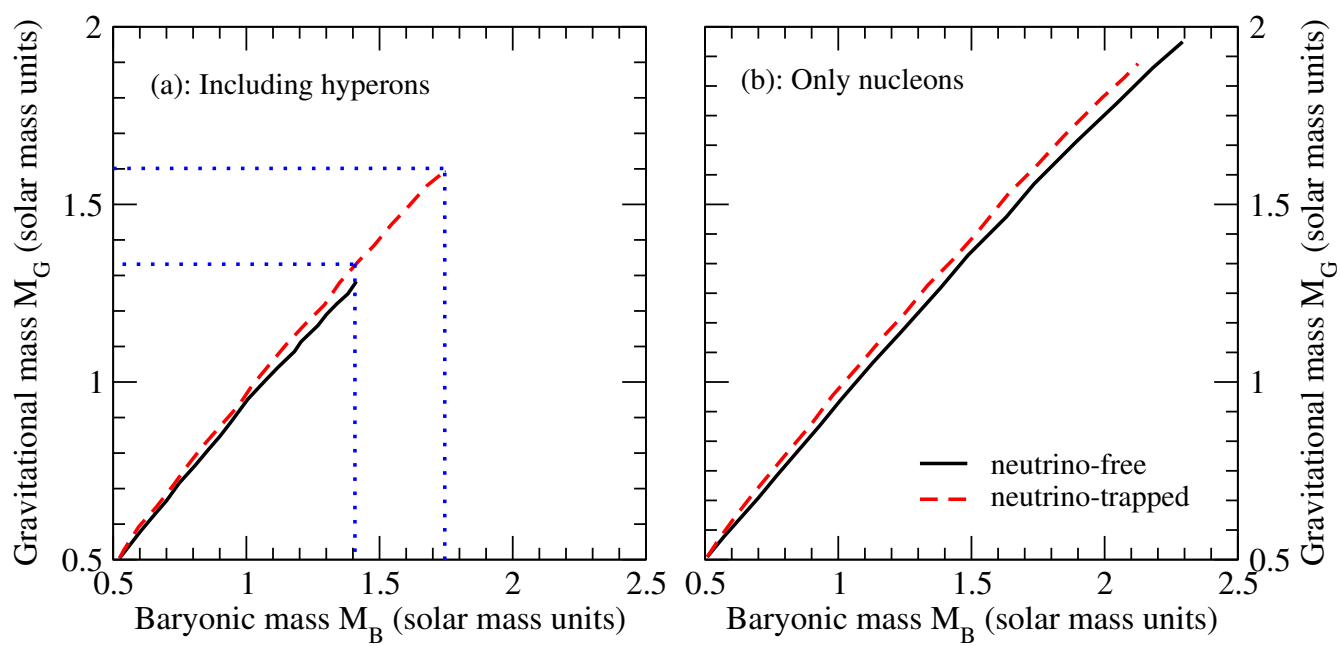

Figure 2: Gravitational mass as a function of the baryonic mass for neutrino-free (solid lines) and neutrinotrapped (dashed lines) matter. Panel (a) shows the results for matter containing nucleons and hyperons, whereas the results for pure nucleonic mater are shown in panel (b). Dotted horizontal and vertical lines show the window of metastability in the gravitational and baryonic masses. Figure adapted from Ref. [45].

hyperons, becomes considerably softer and, consequently, the maximum mass is reduced to values below the current observational limit. Thus, the hyperon puzzle is effectively replaced with the so-called $\Delta$ puzzle.

The possible existence of a Bose-Einstein condensate of negative kaons in the inner core of neutron stars has also been extensively considered in the literature (see e.g., [38, 39, 40, 41, 42, 43] and references therein). As the density of stellar matter increases, the $K^{-}$chemical potential, $\mu_{K^{-}}$, is lowered by the attractive vector meson field originating from dense nucleonic mater. When $\mu_{K^{-}}$becomes smaller than the electron chemical potential $\mu_{e}$ the process $e^{-} \rightarrow K^{-}+v_{e}$ becomes energetically possible. The critical density for this process was calculated to be in the range $2.5-$ $5 \rho_{0}[41,42]$. However, as in the case of the $\Delta$, the appearance of the kaon condensation induces also a strong softening of the EoS and consequently leads to a reduction of the maximum mass to values below the current observational limits. The interested reader is referred to the original work on this subject [38, 39, 40, 41, 42, 43] for a comprehensive description of the implications of kaon condensation on the structure and evolution of neutron stars.

\section{Hyperon stars at birth and neutron star cooling}

As pointed out in the introduction, neutron stars are formed in Type-II, Ib or Ic supernova explosions. The properties of newly born neutron stars are affected by the thermal effects and neutrino trapping. These two effects have a strong influence on the overall stiffness of the EoS and the composition of the star. In particular (see e.g., $[44,45,46]$ ) matter becomes more proton rich, the number of muons is significantly reduced, and the onset of hyperons is shifted to higher densities. In addition, the number of strange particles is on average smaller and the EoS is stiffer in comparison with the cold and neutrino-free case. 
A very important implication of neutrino trapping in dense matter is the possibility of having metastable neutron stars and a delayed formation of a "low-mass" $\left(M=1-2 M_{\odot}\right)$ black hole. This is illustrated in Fig. 2 for the case of a BHF calculation of Ref. [45]. The figure shows the gravitational mass $M_{G}$ of the star as a function of its baryonic mass $M_{B}$. If hyperons are present (panel (a)), then deleptonization lowers the range of gravitational masses that can be supported by the EoS from about $1.59 M_{\odot}$ to about $1.28 M_{\odot}$ (see dotted horizontal lines in the figure). Since most of the matter accretion on the forming neutron star happens in a very early stages after birth $(t<1$ s), to a good approximation the neutron star baryonic mass stays constant during the evolution from the initial proto-neutron star configuration to the final neutrino-free one. Then, for this particular model, proto-neutron stars which at birth have a gravitational mass between $1.28-1.59 M_{\odot}(\mathrm{a}$ baryonic mass between $1.40-1.72 M_{\odot}$ ) will be stabilized by neutrino trapping effects long enough to carry out nucleosynthesis accompanying a Type-II supernova explosion. After neutrinos leave the star, the EoS is softened and it cannot support anymore the star against its own gravity. The newborn star collapses then to a black hole [44]. On the other hand, if only nucleons are considered to be the relevant baryonic degrees of freedom (panel (b)), no metastability occurs and a black hole is unlikely to be formed during the deleptonization since for each initial neutrino-trapping configuration there exist always a final stable neutrino-free one. If a black hole were to form from a star with only nucleons, it is much more likely to form during the post-bounce accretion stage.

The cooling of the newly born hot neutron stars is driven first by the neutrino emission from the interior, and then by the emission of photons at the surface. Neutrino emission processes can be divided into slow and fast processes depending on whether one or two baryons participate. The simplest possible neutrino emission process is the so-called direct Urca process $\left(n \rightarrow p+l+\bar{v}_{l}\right.$, $\left.p+l \rightarrow n+v_{l}\right)$. This is a fast cooling mechanism which however is only possible when the proton fraction exceeds a critical value $x_{D U R C A} \sim 11 \%$ to $15 \%$ [47], being not possible to conserve the momentum and energy simultaneously for lower proton fractions. Other neutrino processes which lead to medium or slow cooling scenarios, but are operative at any density and proton fraction, are the so-called modified Urca processes $\left(N+n \rightarrow N+p+l+\bar{v}_{l}, N+p+l \rightarrow N+n+v_{l}\right)$, the bremsstrahlung $(N+N \rightarrow N+N+v+\bar{v})$, or the Cooper pair formation $(n+n \rightarrow[n n]+v+\bar{v}, p+$ $p \rightarrow[p p]+v+\bar{v})$, this last operating only when the temperature of the star drops below the critical temperature for neutron superfluidity or proton superconductivity. If hyperons are present in the neutron star interior new neutrino emission processes, like e.g., $Y \rightarrow B+l+\bar{v}_{l}$, may occur providing additional fast cooling mechanisms. Such additional rapid cooling mechanisms, however, can lead to surface temperatures much lower than that observed, unless they are suppressed by hyperon pairing gaps. Therefore, the study of hyperon superfluidity becomes of particular interest since it could play a key role in the thermal history of neutron stars. While the presence of superfluid neutrons in the inner crust of neutron stars, and superfluid neutrons together with superconducting protons in their quantum fluid interior is well established and has been the subject of many studies, there have been very few studies of hyperon pairing, see Refs. [48].

\section{Hyperons and the r-mode instability of neutron stars}

It is well known that the upper limit on the rotational frequency of a neutron star is set by its Kepler frequency $\Omega_{\text {Kepler }}$, above which matter is ejected from the star's equator [49]. However, a 
neutron star may be unstable against some perturbations which prevent it from reaching rotational frequencies as high as $\Omega_{\text {Kepler }}$, setting, therefore, a more stringent limit on its rotation [50]. Many different instabilities can operate in a neutron star. Among them, the so called r-mode instability [51], a toroidal mode of oscillation whose restoring force is the Coriolis force, is particularly interesting. This oscillation mode leads to the emission of gravitational waves in hot and rapidly rotating neutron stars though the Chandrasekhar-Friedman-Schutz mechanism [52]. Gravitational radiation makes an $\mathrm{r}$-mode grow, whereas viscosity stabilizes it. Therefore, an $\mathrm{r}$-mode is unstable if the gravitational radiation driving time is shorter than the damping time due to viscous processes. In this case, a rapidly rotating neutron star could transfer a significant fraction of its rotational energy and angular momentum to the emitted gravitational waves. These waves can provide invaluable information on the internal structure of the star and thus put constraints on the EoS. The recent direct detection of gravitational waves emitted by a merger of two black holes by the LIGO collaboration [53] strengthens the perspectives of studying neutron stars in the gravitational wave spectrum including via astro-seismology.

The bulk $(\xi)$ and the shear $(\eta)$ viscosities provide the main mechanisms for the dissipation of r-modes as well as other oscillation modes in neutron stars. The bulk viscosity is the dominant one at high temperatures $\left(T>10^{9} \mathrm{~K}\right)$ and, therefore, it is important for hot young neutron stars. It is produced when the pulsation modes induce variations in pressure and density that drive the star away from $\beta$-equilibrium. As a result, energy is dissipated as the weak interaction tries to reestablish the equilibrium. In the absence of hyperons or other exotic components, the bulk viscosity of neutron star matter is mainly determined by the reactions of direct and modified Urca processes. However, as soon as hyperons appear new mechanisms such as weak non-leptonic hyperon reactions $(N+N \leftrightarrow N+Y, N+Y \leftrightarrow Y+Y)$, hyperonic direct Urca $\left(Y \rightarrow B+l+\bar{v}_{l}, B+l \rightarrow Y+v_{l}\right)$ and modified Urca $\left(B^{\prime}+Y \rightarrow B^{\prime}+B+l+\bar{v}_{l}, B^{\prime}+B+l \rightarrow B^{\prime}+Y+v_{l}\right)$ processes, or strong interactions $(Y+Y \leftrightarrow N+Y, N+\Xi \leftrightarrow Y+Y, Y+Y \leftrightarrow Y+Y)$ contribute to the bulk viscosity and dominate it for $\rho>2-3 \rho_{0}$. For more details on the role of hyperons in damping stellar oscillations via bulk viscosity see Refs. [54].

The time dependence of an r-mode oscillation is given by $e^{i \omega t-t / \tau}$, where $\omega$ is the frequency of the mode, and $\tau$ is an overall time scale of the mode which describes both its exponential growth due to gravitational wave emission as well as its decay due to viscous damping. It can be written as $1 / \tau(\Omega, T)=-1 / \tau_{G W}+1 / \tau_{\xi}+1 / \tau_{\eta}$, where $\tau_{G W}$ is the characteristic time-scale associated with emission of gravitational waves, $\tau_{\xi}$ is related to the damping via bulk viscosity, and $\tau_{\eta}$ via shear viscosity. If $\tau_{G W}$ is shorter than both $\tau_{\xi}$ and $\tau_{\eta}$ the mode will exponentially grow, whereas in the opposite case it will be damped away. For each star at a given temperature $T$ one can define a critical angular velocity $\Omega_{c}$ as the smallest root of the equation $1 / \tau\left(\Omega_{c}, T\right)=0$. This equation defines the boundary of the so-called r-mode instability region. A star will be stable against the $\mathrm{r}$-mode instability if its angular velocity is smaller than its corresponding $\Omega_{c}$. On the contrary, a star with $\Omega>\Omega_{c}$ will develop an instability that will cause a rapid loss of angular momentum through gravitational radiation until its angular velocity falls below the critical value. Panel (a) of Fig. 3 shows the r-mode instability region for a pure nucleonic (black solid line) and a hyperonic (red dashed line) star with $M=1.27 M_{\odot}$ [55]. The contributions to the bulk viscosity from direct and modified nucleonic Urca processes as well as from the weak non-leptonic process $n+n \leftrightarrow p+\Sigma^{-}$ included in the calculation are shown in the panel (b) of the figure. It is clearly seen that the widow 

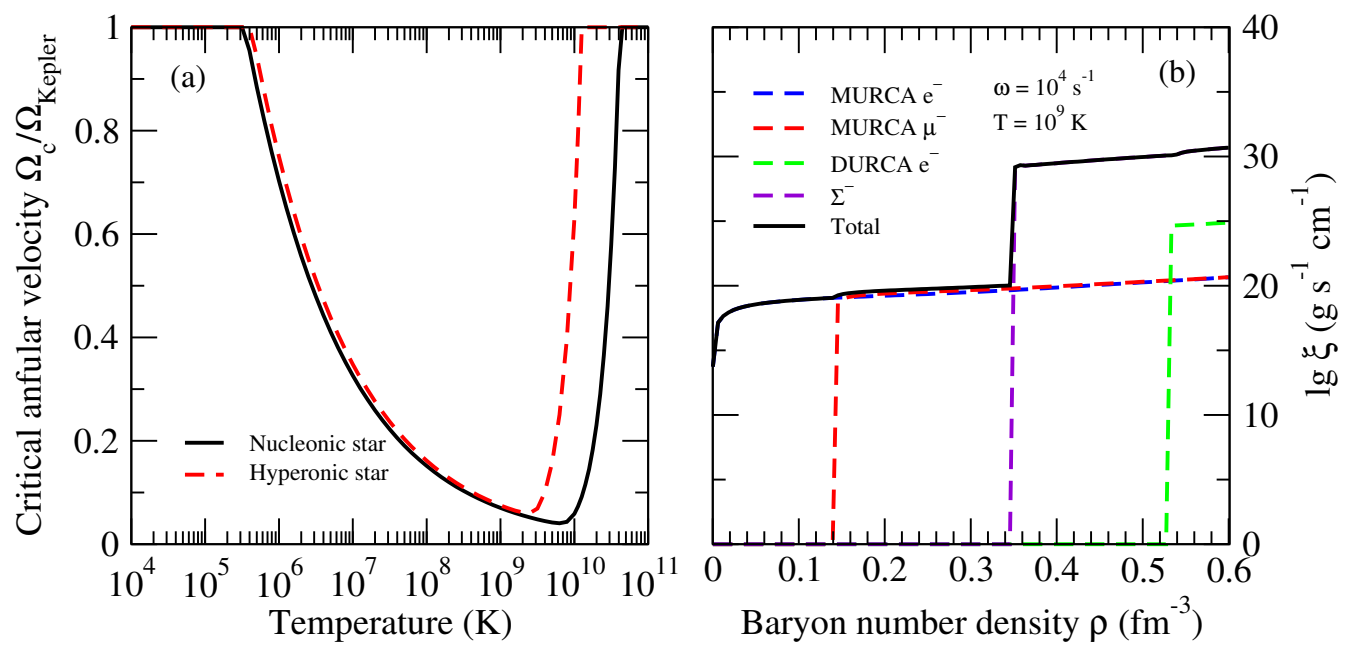

Figure 3: Panel (a): r-mode instability region for a pure nucleonic and a hyperonic star with $1.27 M_{\odot}$. The frequency of the mode is taken as $\omega=10^{4} \mathrm{~s}^{-1}$. Panel (b): Bulk viscosity as a function of the density for $T=10^{9} \mathrm{~K}$ and $\omega=10^{4} \mathrm{~s}^{-1}$. Contributions direct and modified nucleonic Urca processes as well as from the weak non-leptonic process $n+n \leftrightarrow p+\Sigma^{-}$are included. Figure adapted from Ref. [55].

of the r-mode instability is smaller for hyperonic stars, which is the direct consequence of the fact that the bulk viscosity of hyperon rich matter is larger than for purely nucleonic matter and, therefore, the damping in the former case is more effective.

\section{Summary}

In this review article I discussed the main effects of hyperons on the properties of neutron stars with an emphasis on the so-called "hyperon puzzle", i.e., the problem of the strong softening of the EoS of dense matter due to the appearance of hyperons which leads to maximum masses of compact stars that are not compatible with the recent observations of $\sim 2 M_{\odot}$ millisecond pulsars. I have discussed three different solutions proposed to tackle this problem: (i) more repulsion in hyperon-hyperon interactions within the density functional theories of hypernuclear matter in the vector and/or scalar mesons exchange channels; (ii) repulsive hyperonic three-body forces in the ab initio microscopic calculations, and (iii) a phase transition to deconfined quark matter at densities below the hyperon threshold. I have also presented a discussion of how the presence of hyperons will affect the cooling of neutron stars and the r-mode instability window through modifications of microscopic input of weak interaction rates and transport coefficients of dense matter.

\section{Acknowledgements}

The author is grateful to the organizers of MPCS 2015 for their invitation. This work is partly supported by the project PEst-OE/FIS/UI0405/2014 developed under the initiative QREN financed by the UE/FEDER through the program COMPETE-"Programa Operacional Factores de Competitividade", and by "NewCompstar", COST Action MP1304. 


\section{References}

[1] S. L. Shapiro and S. A. Teukolsky, Black Holes, White Dwarf and Neutron Stars (Wiley, New York) 1983; N. K. Glendenning, Compact Stars: Nuclear Physics, Particle Physics and General Relativity, 2nd edition (Springer, Berlin) 2000; P. Haensel, A. Y. Potekhin and D. G. Yakovlev, Neutron Stars 1: Equation of State and Structure, Astrophysics and Space Science Library Series, Vol. 326 (Springer, New York) 2007.

[2] V. A. Ambartsumyan and G. S. Saakyan, Sov. Astron. 4, 187 (1960).

[3] N. K. Glendenning, Phys. Lett. B 114, 392 (1982); N. K. Glendenning, Astrophys. J. 293, 470 (1985); N. K. Glendenning, Z. Phys. A 326, 57 (1987); N. K. Glendening and S. A. Moszkowski, Phys. Rev. Lett. 67, 2414 (1991); F. Weber and M. K. Weigel, Nucl. Phys. A 505, 779 (1989). R. Knorren, M. Prakash and P. J. Ellis, Phys. Rev. C 52, 3470 (1995); J. Schaffner and I. Mishustin, Phys. Rev. C 53, 1416 (1996); H. Huber, F. Weber, M. K. Weigel and Ch. Schaab, Int. J. Mod. Phys. E 7, 310 (1998).

[4] S. Balberg and A. Gal, Nucl. Phys. A 625, 435 (1997); S. Balberg, I. Lichtenstadt and G. B. Cook, Astrophys. J. Suppl. Ser. 121, 515 (1999). D. E. Lanskoy and Y. Yamamoto, Phys. Rev. C 55, 2330 (1997); T. Y. Tretyakova and D. E. Lanskoy, Eur. Phys. J. A 5, 391 (1999); J. Cugnon, A. Lejeune and H.-J. Schulze, Phys. Rev. C 62, 064308 (2000); I. Vidaña, A. Polls, A. Ramos and H.-J. Schulze, Phys. Rev. C 64, 044301 (2001); X.-R. Zhou, H.-J. Schulze, H. Sagawa, C.-X. Wu and E.-G. Zhao, Phys. Rev. C 76, 034312 (2007); X.-R. Zhou, A. Polls, H.-J. Schulze and I. Vidaña, Phys. Rev. C 78, 054306 (2008).

[5] H.-J. Schulze, M. Baldo, U. Lombardo, J. Cugnon and A. Lejeune, Phys. Lett. B 35521 (1995); H.-J. Schulze, M. Baldo, U. Lombardo, J. Cugnon and A. Lejeune, Phys. Rev. C 57, 704 (1998); M. Baldo, G. F. Burgio and H.-J. Schulze, Phys. Rev. C 58, 3688 (1998). M. Baldo, G. F. Burgio and H.-J. Schulze, Phys. Rev. C 61, 055801 (2000); I. Vidaña, A. Polls, A. Ramos, M. Hjorth-Jensen and V. G. J. Stoks, Phys. Rev. C 61, 025802 (2000); I. Vidaña, A. Polls, A. Ramos, L. Engvik and M. Hjorth-Jensen, Phys. Rev. C 62, 035801 (2000); H-J. Schulze, A. Polls, A. Ramos and I. Vidaña, Phys. Rev. C 73, 058801 (2006); H.-J. Shulze and T. Rijken, Phys. Rev. C 84, 035801 (2011).

[6] H. Dapo, B.-J. Schaefer and J. Wambach, Phys. Rev. C 81, 035803 (2010).

[7] F. Sammarruca, Phys. Rev. C 79034301 (2009).

[8] T. Katayama and K. Saito, arXiv:1410.7166 (2014); arXiv:1501.05419 (2015).

[9] D. Lonardoni, F. Pederiva and S. Gandolfi, Phys. Rev. C 89014314 (2014).

[10] P. M. M. Maessen, T. A. Rijken and J. J. de Swart, Phys. Rev. C 40, 2226 (1989); Th. A. Rijken, V. G. J. Stoks and Y. Yamamoto, Phys. Rev. C 59, 21 (1999); V. G. J. Stoks and Th. Rijken, Phys. Rev C 59, 3009 (1999); Th. A. Rijken, Phys. Rev. C 73, 044007 (2006); Th. A. Rijken and Y. Yamamoto, Phys. Rev. C 73, 044008 (2006).

[11] B. Holzenkamp, K. Holinde and J. Speth, Nucl. Phys. A 500, 485 (1989); J. Haidenbauer and U.-G. Meissnes, Phys. Rev. C 72, 044005 (2005).

[12] H. Polinder, J. Haidenbauer and U.- G. Meissner, Nucl. Phys. A 779, 244 (2006); J. Haidenbauer, S. Petschauer, N. Kaiser, U.-G. Meissner, A. Nogga and W. Weise W 2013 Nucl. Phys. A 91524.

[13] Nuclear Methods and Nuclear Equation of State (International Review of Nuclear Physics, Vol 8. Edited by Marcello Baldo. World Scientific Publishing Company 1999); H. Müther and A. Polls, Prog. Part. Nucl. Phys. 45, 243 (2000).

[14] R. A. Hulse and J. H. Taylor, Astrophys. J. Lett. 195 L51 (1975). 
[15] D. J. Champion et al., Science 320, 1309 (2008); P. C. C. Freire, arXiv:0907.32199; arXiv:1011.5809.

[16] P. Demorest et al., Nature 467, 1081 (2010).

[17] J. Antoniadis et al., Science 3406131 (2013).

[18] I. Bednarek, P. Haensel, J. L. Zdunik, M. Bejger and R. Mańka, Astron. and Astrophys. 543, A157 (2012).

[19] S. Weissenborn, D. Chatterjee, and J. Schaffner-Bielich, Phys. Rev. C 85, 065802 (2012).

[20] M. Oertel, C. Providência, F. Gulminelli and Ad. R. Raduta, J. Phys. G. 42075202 (2015).

[21] K. A. Maslov, E. E. Kolomeitsev and D. N. Voskresensky, Phys. Lett. B 748369 (2015).

[22] E. N. E. van Dalen, G. Colucci and A. Sedrakian, Phys. Lett. B 734, 383 (2014).

[23] T. Takatsuka et al., Eur. Phys. J. A 13, 213 (2002); Prog. Theor. Phys. Suppl. 174, 80 (2008).

[24] I. Vidaña, D. Logoteta, C. Providência, A. Polls and I. Bombaci, Eur. Phys. Lett. 94, 11002 (2011).

[25] Y. Yamamoto, T. Furumoto, B. Yasutake and Th. A. Rijken, Phys. Rev. C 88022801 (2013); Phys. Rev. C 90045805 (2014).

[26] D. Lonardoni, A. Lovato, S. Gandolfi and F. Pederiva Phys. Rev. Lett. 114092301 (2015).

[27] M. Baldo, M. Buballa, G. F. Burgio, F. Neumann, M. Oertel and H.-J. Schulze, Phys. Lett. B 562, 153 (2003).

[28] M. Baldo, G. F. Burgio, P. Castorina, S. Plumari and Z. Zappalà, Phys. Rev. C 75, 035804 (2007).

[29] A. Kurkela, P. Romatschke and A. Vuorinen, Phys. Rev. D 81, 105021 (2010).

[30] F. Özel, D. Psaltis, S. Ransom, P. Demorest and M. Alford, Astrophys. J. Lett. 724 L199 (2010).

[31] S. Weissenborn, I. Sagert, G. Pagliara, M. Hempel and J. Schaeffner-Bielich, Astophys. J. Lett. 740 L14 (2011).

[32] T. Klähn, D. Blaschke and D. Lastowiecki, Phys. Rev. D 88085001 (2013).

[33] L. Bonanno and A. Sedrakian, Astron. and Astrophys. 539416 (2012).

[34] R. Lastowiecki, D. Blaschke, H. Grigorian and S. Typel Acta Phys. Polon. Suppl., 5535 (2012).

[35] O. Hashimoto and H. Tamura, Prog. Part. Nucl. Phys. 57564 (2006).

[36] J. L. Zdunik and P. Haensel, Astron. and Astrophys. 551 A61 (2013).

[37] A. Drago, A. Lavagno, G. Pagliara and D. Pigato, Phys. Rev. C 90, 065809 (2014); EPJ Web Conf. 95, 01011 (2015).

[38] D. B. Kaplan and A. E. Nelson, Phys. Lett. B 175, 57 (1986).

[39] D. B. Kaplan and A. E. Nelson, Phys. Lett. B 179, 409, Erratum (1986).

[40] G. E. Brown, C.-H. Lee, M. Rho and V. Thorsson, Nucl. Phys. A 567, 937 (1994).

[41] V. Thorsson, M. Prakash and J. M. Lattimer, Nucl. Phys. A 572, 693 (1994).

[42] C.-H. Lee, Phys. Rep. 275, 255 (1996).

[43] N. K. Glendenning and J. Schaffner-Bielich, Phys. Rev. Lett. 81, 4564 (1998). 
[44] W. Keil and H.-Th. Janka, Astron. and Astrophys. 296, 145 (1996); I. Bomaci, Astron. and Astrophys. 305, 871 (1996); M. Prakash, I. Bombaci, M. Prakash, P. J. Ellis, R. Knorren, J. M. Lattimer, Phys. Rep. 280, 1 (1997).

[45] I. Vidaña, I. Bombaci, A. Polls and A. Ramos, Astron. and Astrophys. 399, 687 (2003).

[46] G. F. Burgio, H.-J. Schulze and A. Li, Phys. Rev. C 83, 025804 (2011).

[47] J. M. Lattimer, C. J. Pethick, M. Prakash and P. Haensel, Phys. Rev. Lett. 66, 2701 (1991).

[48] S. Balberg and N. Barnea, Phys. Rev. C 57, 409 (1998); T. Takatsuka and R. Tamagaki, Prog. Theor. Phys. 102, 1043 (1999); T. Takatsuka, S. Nishizaki, Y. Yamamoto and R. Tamagaki, Prog. Theor. Phys. 105, 179 (2000); T. Takatsuka, S. Nishizaki, Y. Yamamoto and R. Tamagaki, Prog. Theor. Phys. Suppl. 146, 279 (2002); I. Vidaña and L. Tolós, Phys. Rev. C 70, 028802 (2004); X.-R. Zhou, H.-J. Schulze, F. Pan and J. P. Drayer, Phys. Rev. Lett. 95, 051101 (2005); Y. N. Wag and H. Shen, Phys. Rev. C 81, 025801 (2010).

[49] L. Lindblom, Astrophys. J. 303, 146 (1986); J. L. Friedman, J. R. Ipser and L. Parker, Astrophys. J. 304, 115 (1986).

[50] L. Lindblom, Astrophys. J. 438, 265 (1995).

[51] N. Anderson, Astrophys. J. 502, 708 (1998); J. L. Friedman and S. M. Morsink, Astrophys. J. 502714 (1998).

[52] S. Chandrasekhar, Phys. Rev. Lett. 24, 611 (1970); J. L. Friedman and B. F. Schutz, Astrophys. J. 221, 937 (1978); J. L. Friedman and B. F. Schutz, Astrophys. J. 222, 281 (1978); J. L. Friedman, Commun. Math. Phys. 62, 247 (1978).

[53] B. P. Abbott et al. (LIGO Scientific Collaboration and Virgo Collaboration), Phys. Rev. Lett. 116, 061102

[54] W. D. Langer, and A. G. W. Cameron, Astrophys. Space Sci. 5213 (1969); P. B. Jones, Proc. R. Soc. A 323, 111 (1971); Y. Levin, Astrophys. J. 517, 328 (1999); P. B. Jones, Phys. Rev. Lett. 86, 1384 (2001); P. B. Jones, Phys. Rev. D 64, 084003 (2001); L. Lindblom and B. J. Owen, Phys. Rev. D 65, 0653006 (2002); P. Haensel, K. P. Levenfish, and D. G. Yakovlev, Astron. and Astrophys. 381, 1080 (2002); E. N. E. van Dalen, and A. E. Dieperink, Phys. Rev. C 69, 025802 (2004); D. Chatterjee, and D. Bandyopadhyay, Phys. Rev. D 74, 023003 (2006); R. Bondarescu, S. A. Teukolsky, and I. Wasserman, Phys. Rev. D 76, 064019 (2007); D. Chatterjee, and D. Bandyopadhyay Astrophys. J. 680, 686 (2008); M. E. Gusakov, and E. M. Kantor, Phys. Rev. D 78, 083006 (2008); M. Sinha, and D. Bandyopadhyay, Phys. Rev. D 79, 123001 (2009); A. Patruno, Astrophys. J. 722, 909 (2010); T. K. Jha, H. Mishra, and V. Sreekanth, Phys. Rev. C 82, 025803 (2010).

[55] I. Vidaña and C. Albertus, in preparation (2016). 